\title{
« LA COULEUR DU TRAVAIL » : UNE ANTHROPOLOGIE HISTORIQUE DE LA FABRIQUE DE LA RACE DANS LA PLANTATION
}

\author{
Entretien avec $\underline{\text { Cristiana Bastos, Propos recueillis par Colette Le Petitcorps, }}$ \\ Amandine Desille
}

Centre d'Information et d'Etudes sur les Migrations Internationales | "Migrations Société »

2020/4 N 182 | pages 113 à 126

ISSN 0995-7367

Article disponible en ligne à l'adresse :

https://www.cairn.info/revue-migrations-societe-2020-4-page-113.htm

Distribution électronique Cairn.info pour Centre d'Information et d'Etudes sur les Migrations Internationales.

(C) Centre d'Information et d'Etudes sur les Migrations Internationales. Tous droits réservés pour tous pays.

La reproduction ou représentation de cet article, notamment par photocopie, n'est autorisée que dans les limites des conditions générales d'utilisation du site ou, le cas échéant, des conditions générales de la licence souscrite par votre établissement. Toute autre reproduction ou représentation, en tout ou partie, sous quelque forme et de quelque manière que ce soit, est interdite sauf accord préalable et écrit de l'éditeur, en dehors des cas prévus par la législation en vigueur en France. Il est précisé que son stockage dans une base de données est également interdit. 


\title{
« La couleur du travail » : une anthropologie historique de la fabrique de la race dans la plantation
}

\section{Entretien avec Cristiana Bastos"}

\author{
Propos recueillis par Colette Le Petitcorps ${ }^{\star \star}$ et Amandine Desille ${ }^{\star \star \star}$
}

\section{RÉSUMÉ}

Cristiana Bastos a mené ses travaux aux États-Unis et au Portugal sur la biopolitique en contexte colonial, l'histoire de la santé, les dynamiques de populations liées aux mobilités transnationales et la production de catégories racialisées. Cet entretien revient sur les origines de son travail conceptuel en mettant l'accent sur les découvertes empiriques qui l'ont conduite à repenser les liens entre les migrations portugaises et la colonisation. Sont évoquées les expériences de terrain qui l'ont détournée de l'étude des colons dans l'empire portugais, pour explorer plutôt les vies des travailleurs engagés portugais, pris dans les flux transimpériaux de main-d'œuvre vers les économies de plantation telles le Guyana ou Hawaï. L'originalité de sa contribution au champ de recherches anthropologiques et historiques sur les plantations réside dans l'articulation de plusieurs régimes d'exploitation de la force de travail importée dans son analyse de la construction sociale des différences, ce qui est rendu explicite par l'étude du cas peu connu des travailleurs engagés portugais. Le projet de recherche ERC Advanced Grant The Colour of Labour. The Racialised Lives of Migrants qu'elle conduit actuellement à l'Université de Lisbonne est l'aboutissement de cette réflexion : celui-ci interroge les racines historiques de la racialisation dans la forme coloniale spécifique de la plantation agricole.

Mots-CLÉs : Angola, colonisation, États-Unis, exploitation du travailleur, Guyana, Hawaï, migration de main-d'œuvre, Portugais, Portugal

Amandine Desille et Colette Le Petitcorps: En 2005, tu découvres au sud de I'Angola la tombe d'une femme, Maria Índia, morte en 1938. Ta promenade fortuite dans un cimetière devient le point de départ de la reconstitution d'une histoire effacée par la narration impérialiste portugaise. Peux-tu raconter I'histoire de ta rencontre avec Maria Índia

* Professeure en anthropologie, coordinatrice de l'ERC AdG 2015 - 695573 The Colour of Labour, ICS, Université de Lisbonne.

** Sociologue, chercheuse postdoctorante au sein de l'ERC AdG 2015 - 695573 The Colour of Labour, PI Cristiana Bastos, ICS, Universidade de Lisboa.

*** Chercheuse postdoctorante, IGOT, Universidade de Lisboa. Les recherches menant aux présents résultats ont bénéficié d'un soutien financier du septième programme-cadre de I'Union européenne (7ePC/2007-2013) en vertu de la convention de subvention $n^{\circ} 794030$, pour le projet intitulé MigRural. 
et ce qu'elle t'a permis de révéler de l'histoire complexe des migrations portugaises du temps des colonies?

Cristiana Bastos: Cela a été un heureux moment de découverte qui a ouvert la voie à d'autres trouvailles dont l'assemblage $\mathrm{m}^{\prime}$ 'a conduite à la recherche actuelle. Cela faisait déjà plusieurs années que je tentais d'analyser un épisode de la colonisation européenne sur le plateau de Huíla au sud de l'Angola. J'avais repéré cette poche de colonisation qui était directement reliée à la recherche que je menais à l'époque sur les médecins de Goa. Un médecin particulièrement prolixe était passé par le plateau de Huíla dans les années 1920 et avait mis en pratique ses méthodes d'investigation anthropométrique à propos de ce qu'il appelait la communauté des «Euro-Africains » (eurodescendants en Afrique). Celui-ci utilisa ses observations pour soutenir les théories racialistes, proches du suprémacisme blanc - une doctrine dont nous connaissons les conséquences catastrophiques - qui avait à l'époque ses appuis politiques et qui florissait dans les milieux scientifiques, y compris dans l'anthropologie physique. L'analyse du cas des travaux de ce médecin que je faisais dans l'article «Um luso-tropicalismo às avessas » (《Un luso-tropicalisme à rebours $\gg)^{1}, \mathrm{~m}^{\prime}$ a permise de mettre en évidence la façon dont le racialisme et le racisme explicites coexistaient bel et bien avec les propositions du luso-tropicalisme. Le lusotropicalisme est une doctrine adoptée par le régime de Salazar qui affirmait que les Portugais n'avaient jamais pratiqué de racisme. Elle s'appuyait sur les idées du sociologue brésilien Gilberto Freyre qui avançait que la nature créolisée des Portugais induisait de fait une interaction moins violente (dite moins raciste) avec les peuples des tropiques que ce qui avait cours avec les autres Européens. L'idée a tellement circulé par les moyens d'information du régime salazariste et dans les écoles qu'il y a des gens, jusqu'à aujourd'hui, qui croient que les Portugais sont moins racistes que les autres colonisateurs ${ }^{2}$.

Mais il y avait autre chose : d'où venait cette communauté des soidisant « euro-africains »? À partir des années 2000, des historiens et

1. BASTOS, Cristiana, "Um luso-tropicalismo às avessas: colonialismo científico, aclimação e pureza racial em Germano Correia", in : FERREIRA, Ana Paula ; RIBEIRO, Margarida Calafate (sous la direction de), Fantasmas e fantasias imperiais no imaginário português contemporâneo, Porto: Campo das Letras, 2003, pp. 227-253.

2. BASTOS, Cristiana, "Tristes trópicos e alegres lusotropicalismos : das notas de viagem em Lévi-Straus e Gilberto Freyre", Análise Social vol. xxxiii, n¹46-147, 1998, pp. 415-432 ; BASTOS, Cristiana, "Luso-Tropicalism Debunked Again: Race, Racism and Racialism in Three Portuguese-Speaking Societies", in:ANDERSON, Warwick; ROQUE, Ricardo ; SANTOS, Ricardo Ventura (eds), Luso-Tropicalism and its discontents: The Making and Unmaking of Racial Exceptionalism, New York: Berghahn, 2019, pp. 243-264 ; CASTELO, Cláudia, O modo português de estar no mundo. O luso-tropicalismo e a ideologia colonial portuguesa (1933-1961), Porto : Afrontamento, 1999, $168 \mathrm{p}$. 
anthropologues dont je fais partie - notons par exemple le groupe de chercheurs brésiliens et portugais ayant contribué à l'ouvrage Trânsitos Coloniais $^{3}$ - ont ouvert un grand débat sur la colonisation portugaise en Afrique en soulignant notamment ses discontinuités et son caractère tardif : cela allait tout à fait à l'encontre de l'idée d'une architecture solide faite de 500 ans d'occupation, telle qu'elle était propagée par le régime colonial et reprise parfois sans perspective critique par les courants anticolonialistes. Cláudia Castelo ${ }^{4}$ montre notamment que la colonisation en Angola a surtout été entreprise par les Européens au $X X^{\mathrm{e}}$ siècle, et qu'elle ne prend sa forme la plus aboutie qu'après la Seconde Guerre mondiale. Jusqu'à cette période récente de colonisation, l'Angola - dont les frontières ont été définies entre Européens dans le contexte du dépeçage de l'Afrique durant la conférence de Berlin en 1884-1885 - était un vaste territoire comprenant plusieurs sociétés africaines composées de groupes nomades et sédentaires aux langues, aux cultures et aux systèmes économiques divers. Seule une poignée d'Européens habitait sur place. II existait bien depuis longtemps un commerce sur la côte et divers canaux qui, à partir des zones de l'intérieur, alimentaient le trafic d'esclaves à destination des plantations du dénommé «nouveau monde », en particulier du Brésil. Cela a été analysé de façon magistrale par Luiz Felipe Alencastro dans $O$ Trato dos Viventes (Le marché des vivants) ${ }^{5}$. Mais il ne s'agissait pas là d'une «colonie de peuplement», sauf cette expérience du plateau de Huíla qui m'a intriguée et que j'ai étudiée à partir des sources disponibles. Promue par le gouvernement portugais et exécutée en 1884 dans une course contre la montre pour le « partage de l'Afrique », l'opération du plateau de Huíla a procédé au transport de quelques centaines de Madériens par voie maritime vers le port de Moçâmedes (aujourd'hui Namibe). Elle s'est poursuivie en 1885 par l'ascension des montagnes de Chela au moyen des voitures Boers et de leurs bœufs. Là, sur un plateau à 2000 mètres d'altitude, se sont développées des colonies agricoles. Puis, là où d'autres avaient failli, se sont succédées et reproduites avec persévérance les communautés des descendants de ces premiers Madériens. Si ces colons, principalement originaires de Madère, furent conduits vers ce plateau dans le but de fixer les frontières coloniales en Afrique - lesquelles se jouaient davantage entre les puissances européennes plus au nord du Portugal -, il y avait aussi

3. BASTOS, Cristiana ; VALE DE ALMEIDA, Miguel ; FELDMAN-BIANCO, Bella (sous la direction de), Trânsitos coloniais: diálogos críticos luso-brasileiros, Lisboa: Imprensa de Ciências Sociais, 2002, $403 \mathrm{p}$.

4. CASTELO, Cláudia, Passagens para África: o povoamento de Angola e Moçambique com naturais da metrópole, Porto : Afrontamento, 2007, 405 p.

5. DE ALENCASTRO, Luiz Felipe, O trato dos viventes. Formação do Brasil no Atlântico sul, São Paulo: Companhia das Letras, 2000, 525 p. 
dans ce mouvement d'autres intentions, explicitées dans les débats parlementaires antérieurs, mais effacées de la mémoire et de la narration de I'histoire. II s'agissait de créer des alternatives au mouvement migratoire qui conduisait des milliers de Portugais vers des nations et des Empires concurrents, principalement vers la Guyane britannique et Hawaï. J'ai étudié de manière approfondie toute la documentation relative aux premiers colons et aux migrations portugaises vers les autres Empires coloniaux. Et heureusement, il existait deux journaux de bord (du commandant et du médecin) du voyage du bateau Índia qui a transporté les Portugais depuis Funchal (capitale de Madère) vers Moçâmedes en 1884 et 1885. Dans le journal de bord du médecin est répertoriée la naissance d'une enfant, qui fut baptisée Maria Índia en hommage au bateau où elle fut mise au monde. Quand j'ai pu me rendre en Angola pour rechercher plus de sources et évaluer la matérialité de l'expérience de la conquête de la forêt par les pionniers, j'ai eu la chance, en étant incitée à visiter le dénommé cimetière Boer de Humpata, de me trouver nez à nez devant une tombe marquée du nom de Maria Índia. II s'agit d'un nom pourtant rare, et la date de naissance concordait : ça ne pouvait qu'être la même personne. Ce fut un véritable sursaut cognitif que je raconte dans l'article «Maria Índia, ou a fronteira da colonização » (《Maria Índia, ou la frontière de la colonisation $\gg)^{6}$. Le texte manqua de ne pas être publié, parce qu'il s'appuyait sur une conjecture : je ne pouvais pas attester à $100 \%$ qu'il s'agissait de la même personne, et je déduisais beaucoup de choses avec peu de données. Mais à force de révisions et de négociations, l'article fut tout de même publié, et mis immédiatement en ligne en accès libre sur le portail de recherche Scielo. Et à partir de là surgit une autre coïncidence encore plus extraordinaire : I'article fut lu par les descendants de la même Maria Índia, qui ne me connaissaient pas et que je ne connaissais pas, et je reçus soudain des emails d'Afrique du Sud, puis du Brésil et du Portugal. De tous côtés apparaissaient des descendants avec leurs mémoires de famille. La vie de Maria Índia ne se résumait pas à une conjecture, elle était bien une réalité : il y avait des photos, des arbres généalogiques et des histoires de grand intérêt qui me furent partagées. Parmi celles-ci, j'appris que la fille qui était née à bord de I'Índia avait deux frères plus âgés qui étaient nés à Honolulu. Ses parents ne venaient donc pas directement de Madère: ils étaient déjà passés par Hawaï. Ce constat me conduisit à repenser la dynamique des flux migratoires, que j'appréhendais jusque-là uniquement par la dispersion des parcours individuels. J'entrepris d'explorer les superpositions et les connexions de ces mobilités de longue distance, qui

6. BASTOS, Cristiana, "Maria Índia, ou a fronteira da colonização: trabalho, migração e política no planalto sul de Angola", Horizontes Antropológicos, vol. 15, n³1, 2009, pp. 51-74. 
impliquaient surtout les îliens portugais de Madère et des Açores venant de sociétés très stratifiées avec une large population vulnérable. Or, ces migrants empruntaient souvent un chemin, et un autre, et encore un autre, comme je le vérifiais dans les sources documentaires. $\mathrm{J}$ 'ai donc tenté d'engager une recherche combinant cette dyade migrations-Empire en explorant les différentes possibilités conceptuelles.

A. Desille et C. Le Petitcorps : Justement, dans ton article « Portuguese in the Cane ${ }^{7}$, tu dis qu'il n'y a pas eu une expérience conquérante de la navigation portugaise dans le monde, mais plutôt de multiples expériences des mobilités et des re-mobilités de Portugais, en particulier açoriens et madériens, entre les divers espaces coloniaux au cours du $X I X X^{e}$ siècle. Peux-tu préciser les enjeux épistémologiques de ta recherche sur ces mobilités transimpériales pour la déconstruction du récit colonial de l'Empire portugais?

C. Bastos: L'idée d'un Empire continu et ininterrompu qui serait né des découvertes du $X V^{e}$ siècle et consolidé jusqu'à la fin $d u X X^{e}$ siècle, est peu remise en question. Elle a été tellement véhiculée qu'elle a été traduite dans les procédés éducatifs tout au long du XX $X^{e}$ siècle. $Y$ compris les approches les plus critiques, qui ont continué de penser le colonialisme comme une oppression constante et stable durant 500 ans. Les historiens avaient toutefois déjà démontré qu'il existait des cycles et des discontinuités : en général venait le cycle du commerce des épices d'Asie au $\mathrm{XVI}^{\mathrm{e}}$ siècle, suivi du long cycle du commerce transatlantique comprenant l'extraction des ressources naturelles et l'implantation de la monoculture au Brésil avec l'esclavage en masse d'Africains, et enfin la colonisation de l'Afrique que certains (parmi lesquels je ne m'inclus pas) nomment le «troisième Empire». Les historiens ont également montré la diversité des formes des relations politiques dans les lieux de contact, où les Portugais pouvaient dominer ou au contraire être les vassaux, ou les clients, ou les associés, etc. Plusieurs mondes coexistèrent. II y avait des centres administratifs et commerciaux, comme à Goa à l'époque moderne, subordonnés au règne impérial par le biais de vices-rois portugais et d'une masse ecclésiastique et militaire. Mais ces pouvoirs étaient en même temps impliqués dans des négociations avec les structures autochtones, créant des formes inédites de gouvernement qui articulaient les dynamiques du pouvoir local avec le pouvoir central. Il y eut le développement de structures de pouvoir parallèles et contradictoires dans les

7. BASTOS, Cristiana, "Portuguese in the Cane: The Racialization of Labour in Hawaiian Plantations", Changing Societies: Legacies and Challenges, Vol. 1. 2018, pp. 65-96. 
vastes extensions territoriales comme au Brésil, ainsi que des routes de trafic soutenues par le pouvoir central, tandis que d'autres restèrent en-dehors de son contrôle. II y eut des incursions et des expériences aux confins de l'Empire qui jouaient avec les identifications «portugaises » en fonction des circonstances comme le montre António Hespanha $^{8}$; et finalement, pour ce qui nous intéresse le plus, il existait d'innombrables itinéraires de déplacement en-dehors des frontières de l'Empire. Récemment, le spécialiste de I'histoire coloniale portugaise Malyn Newitt ${ }^{9}$ a présenté une approche alternative de I'histoire des Portugais en donnant plus d'importance à leurs parcours migratoires qu'aux missions du pouvoir impérial. C'est un angle d'approche analogue à celui que je me suis efforcée de mobiliser pour interpréter un grand nombre d'éléments qui apparaissent entre les mailles de I'Empire, avec lui, malgré lui et parfois contre lui. Y compris à Moçâmedes (Namibe), où furent importés des colons madériens sous parrainage officiel en 1884, il existait déjà une colonie d'Algarviens, qui étaient arrivés là de leur propre initiative, qui avaient navigué sur des milliers de kilomètres le long de la côte africaine avec leur bateau de pêche, sans avoir aucune relation avec le pouvoir impérial, bien au contraire : ils étaient à la marge de l'Empire. La pêche au moyen et long cours ainsi que la chasse à la baleine ont créé des itinéraires de mobilité qui ont conduit à de nombreux déplacements, qui ne représentaient pas les logiques de l'Empire, mais qui avaient un impact démographique, économique et culturel considérable. La chasse à la baleine a entraîné des Polynésiens vers l'Atlantique et vice-versa avec les Portugais des îles qui accostaient leurs bateaux en Polynésie pour le ravitaillement en eau et en produits frais. Quand les Portugais ont commencé à aller à Hawaï pour travailler dans les plantations sucrières en 1878, il existait déjà dans l'archipel une petite colonie de près de 400 Madériens, Açoriens et Capverdiens arrivés là par les baleiniers quelques décennies auparavant. Certains avaient embarqué depuis leur propre île, tandis que d'autres venaient du grand centre baleinier de l'époque, New Bedford dans le Massachusetts, où affluaient les équipages des flottes du monde entier. La Nouvelle-Angleterre devint aussi une destination quasi incontournable des routes prises par les îliens portugais, d'abord marins, puis travailleurs migrants de l'industrie textile. II s'y forma l'une des plus grandes «colonies » portugaises en termes démographiques, économiques et culturels, également distincte et contradictoire du projet impérial portugais. Quant aux histoires des

8. HESPANHA, António, Filhos da Terra: Identidades Mestiças nos Confins da Expansão Portuguesa, Lisboa : Tinta-da-China, 2019, 368 p.

9. NEWITT, Malyn Dudley Dunn, Emigration and the Sea: An Alternative History of Portugal and the Portuguese, Oxford: Oxford University Press, 2015, 256 p. 
individus et des familles qui accomplirent de multiples périples, j'ai quelques exemples de cas qui combinent Hawaï et I'Angola, le Guyana et Hawaï, la Nouvelle-Angleterre et Hawaï, etc., mais les données demeurent limitées. La quasi-inexistence de témoignages personnels écrits, la rareté des journaux de bord pour les trajets entre Madère et le Guyana, Madère et Hawaï, les Açores et Hawaï, rend la tâche sinon impossible, du moins morose. L'ouvrage A Tale of Two Plantations dans lequel Richard Dunn ${ }^{10}$ compare minutieusement la vie sociale et l'économie d'une plantation de Jamaïque avec celle d'une plantation de Virginie, en suivant les histoires de beaucoup d'esclaves et d'affranchis qui y travaillaient à partir de listes nominatives, fut le travail de plusieurs décennies de compilation et d'analyse. J'espère ne pas prendre encore trop de temps dans cette modeste aventure, qui durera encore un peu néanmoins. Entre-temps, du fait du manque de documents personnels ou de récits de voyage, je suis en train de travailler avec la documentation qui concerne l'installation dans les lieux de migration et bien sûr, avec les histoires orales relatives aux mémoires de famille. J'inclus dans I'analyse les trajectoires socioéconomiques de la plantation vers la ville, de la servitude vers le commerce et l'exploitation d'élevage, etc., et je considère aussi les déplacements géographiques secondaires qui créent parfois de nouvelles trajectoires, d'Hawaï vers la Californie, ou du Guyana vers le Canada par exemple.

A. Desille et C. Le Petitcorps: Comment ces histoires migratoires de ressortissants portugais entre les Empires ou dans les marges de I'Empire colonial portugais t'ont mise sur la piste de la proposition théorique avancée dans ton projet de recherche actuel, The Colour of Labour. The Racialised Lives of Migrants?

C. Bastos : Ce fut tout un cheminement comportant quelques expérimentations théoriques et méthodologiques, en gardant comme noyau central de référence empirique les voyages des Madériens et des Açoriens en Guyane britannique, à Hawaï et dans le sud de l'Angola, auxquels j'ai ajouté quelques éléments de contexte complémentaires, soit par connexion directe ou pour tester le potentiel analytique de ces expérimentations dans des contextes totalement différents. Le premier objectif était de combler le fossé entre les études impériales/coloniales et les études migratoires, qui coexistent séparément, forment des spécialistes différents qui ne dialoguent pas toujours entre eux et promeuvent rarement des travaux de recherche ensemble. Nous avons essayé de pro-

10. DUNN, Richard S., A Tale of Two Plantations, Cambridge: Harvard University Press, 2014, $552 \mathrm{p}$. 
mouvoir ce type de dialogue avec le projet Transits Coloniaux (2002), mais le cadre empirique était centré sur l'axe lusophone Brésil-PortugalAfrique. Ça a été une expérience intéressante pour repenser le colonialisme portugais, ça a permis des lectures croisées, mais cela n'a évidemment pas changé la division traditionnelle entre les études sur les migrations et les études sur les Empires coloniaux.

Je me suis rendue compte qu'au lieu de vouloir atteindre cet objectif tel quel, il valait mieux l'atteindre en analysant des cas croisant les deux champs d'étude, ce qui nécessitait l'utilisation de nouveaux angles théoriques en parallèle. Comme lorsque nous faisons de l'histoire anthropologique, ou dans toute autre approche transdisciplinaire, ce n'est pas en argumentant sur le besoin de convergence que nous y parvenons, mais en combinant les méthodes, la littérature et les problématisations des deux disciplines.

Entre-temps, il y eut une autre coïncidence. Entre 1998 et 2010, j'ai été professeure invitée à plusieurs reprises à l'Université de Brown, où j'ai pu faire des recherches sur les contextes du Guyana et de Hawaï, peu étudiés au Portugal. J'ai aussi pu en apprendre davantage sur l'histoire, la démographie et la société de Madère et des Açores, ainsi qu'aller directement à la rencontre de la communauté luso-descendante de Rhode Island et du Massachusetts.

J'ai longuement échangé avec l'influent analyste de la condition des Luso-Américains, Onésimo Teotónio de Almeida, professeur à Brown, sur les contrastes entre les communautés luso-descendantes de Nouvelle-Angleterre et de Californie, en termes de dynamiques de mobilité sociale, de stigmatisation, de choix professionnels, etc. Frank de Sousa de l'Université du Massachusetts, m'a aussi invitée à revisiter un livre controversé paru en 1923 sur les communautés portugaises de Nouvelle-Angleterre ${ }^{11}$, ce que j'ai pu faire lors de mon séjour à l'Université du Massachusetts-Dartmouth en 2013 et, quelques mois plus tard, lors de mon séjour à l'Institut Max Planck d'histoire des sciences, avec le groupe de recherche sur les représentations des variations humaines coordonné par Veronika Lipphardt ${ }^{12}$. J'ai analysé la « racialisation » des Portugais, manifeste dans le livre en question et sujette à des critiques immédiates de la part des communautés, qui avaient pourtant ellesmêmes recours à des critères racialisants et même racistes. Je rends compte de cette analyse dans l'article «Migrants, Inequalities and Social Research in the 1920s ( $\ll$ Migrants, inégalités et recherche

11. TAFT, Donald Reed, Two Portuguese Communities in New England, New-York: Columbia University, 1923, $359 \mathrm{p}$.

12. Voir le site internet : https://www.mpiwg-berlin.mpg.de/research/projects/NWGLipphardt. 
sociale dans les années $1920 \gg)^{13}$. Pour faire court, j'ai réalisé à quel point il existait de fortes similitudes entre les processus de « racialisation » - c'est-à-dire la naturalisation hiérarchique des différences qui ont émergé dans les sociétés de plantation (comme dans le Guyana et à Hawaï), dans les colonies (comme au sud de l'Angola) et dans les sociétés industrielles (comme en Nouvelle-Angleterre). Le cas des insulaires portugais s'avérait exemplaire de processus globaux.

Ce qui pour moi avait commencé par un intérêt pour certains itinéraires migratoires entre des empires concurrents, effacés des récits coloniaux, est alors devenu quelque chose de beaucoup plus ambitieux : une proposition théorique sur les dynamiques de racialisation. Le projet The Colour of Labour présente un ensemble de cas montrant que la vie des Portugais s'est davantage jouée dans la dispersion diasporique que dans la construction de l'empire colonial : il souligne que les flux à travers les empires avaient une importance plus démographique et économique que les politiques de colonisation qui pouvaient entrer en concurrence avec eux. Mais à travers ce projet, j'entends surtout contribuer au développement conceptuel de l'ensemble plantation-travail-racialisation et à l'examen de la production de catégories raciales.

A. Desille et C. Le Petitcorps: Quels sont les angles morts que tu repères dans certains travaux sur le racisme, à partir de ton analyse des migrations portugaises dans les divers empires coloniaux au XIX siècle?

C. Bastos: Le problème principal est que de nombreuses œuvres s'enlisent dans la réification de la « race » et ne prêtent pas suffisamment attention aux modes par lesquels elle est produite, reproduite et méta-produite. C'est un débat qui vient de loin: Sydney Mintz luimême, auteur de la préface de l'édition 2010 de The Plantation d'Edgar Thompson $^{14}$ écrite des décennies auparavant, a émis des réserves quant à la diffusion de l'idée d'une «production sociale de la race » parce qu'elle pourrait éclipser la matérialité brutale du racisme. Mais il n'y a rien de dématérialisant dans l'analyse du processus de production d'une catégorie mentale qui a justifié et multiplié tant d'abus physiques, émotionnels et sociaux, qui a naturalisé les hiérarchies associées à un mode de production spécifique (celui de la plantation), qui s'est collée à la peau, à la vision, à l'idéologie, à la science, et qui réapparaît

13. BASTOS, Cristiana, "Migrants, Inequalities and Social Research in the 1920s: The Story of Two Portuguese Communities in New England", History and Anthropology, Vol. 28, No. 2, 2018, pp. 163-183.

14. THOMPSON, Edgar Tristram, The Plantation, Columbia: University of South Carolina Press, 2010 ( $1^{\text {ère }}$ éd. 1932), $176 \mathrm{p}$. 
dans le mirage égalitaire, jamais analytiquement résolu, des ethnicités. Bien sûr, aucun processus de racialisation n'est aussi extrême que celui qui a été appliqué aux Africains réduits en esclavage et victimes de la traite dans les plantations des Amériques. C'est le cas archétypal, dont les autres constituent des formes plus diluées, créées dans des contextes juridiques différents, dans des périodes historiques plus courtes, et dans un registre qui n'a pas impliqué la déshumanisation complète des sujets, bien que des formes et des rythmes de travail extrêmes, des châtiments corporels et la disqualification aient été conservés pour tous les groupes auxquels ont été attribuées des caractéristiques « raciales ». Ce fut le cas à grande échelle pour les travailleurs « engagés», métaphorisés par le terme de «coolie», une catégorie qui fut d'abord utilisée pour désigner les Asiatiques engagés en général, puis restreinte aux Asiatiques du Sud qui dans l'Empire britannique furent déplacés vers les plantations de l'île Maurice dans l'Océan Indien, de Guyane et de Trinidad dans les Caraïbes, ainsi que des Fidji dans le Pacifique. Et c'est ce qui s'est passé dans d'autres situations, avec les Mélanésiens kidnappés (blackbirding) dans les plantations de Fidji ou d'Australie, ou encore avec les Japonais, les Portugais ou les Philippins engagés pour Hawaï. Et, dans un autre registre, les migrants européens qui sont venus par vagues successives alimenter la main-d'œuvre nécessaire à la révolution industrielle nord-américaine ont également été racialisés, au cours d'un processus de substitution rapide des nouveaux groupes assimilés à la majorité par d'autres primo arrivants. Cela a été montré à propos des Irlandais, des Juifs et des Italiens aux États-Unis. Et cela s'est produit aussi, plus tard et sur une période de migrations vers les États-Unis plus longue, avec les Portugais.

Dans les sociétés résultant de racialisations multiples, où au lieu de la polarité noir/blanc de la plantation originelle coexistent de multiples groupes, il y en a qui se bercent dans l'illusion d'une multi-ethnicité harmonieuse, quand ce n'est pas pour y trouver un paradis de relations raciales comme on le voit dans les descriptions du Hawaï des années 1930 faites par les sociologues de l'École de Chicago. À l'opposé de ces approches se trouvent des analyses comme celle de Brackette Williams $^{15}$ sur le Guyana post-indépendance, qui soulignent l'effet écrasant de la production continue de catégories de population en tension et en compétition dans le récit historique de la construction de la nation jusqu'à aujourd'hui, comme cela est le cas entre les afrodescendants et les indo-descendants.

15. WILLIAMS, Brackette F, Stains on my Name, War in my Veins: Guyana and the Politics of Cultural Struggle, Durham: Duke University Press, 1991, 342 p. 
En bref, si le cœur du processus de racialisation se trouve dans la société de plantation, il se prolonge et se poursuit dans une dynamique alimentée par le racisme, qui le reproduit et l'amplifie. Le racisme, avec son mécanisme sous-jacent de hiérarchisation, perpétue la racialisation y compris là où on s'y attend le moins. Je dirais que c'est l'angle mort que j'aimerais le plus souligner : la dynamique de la production et de la reproduction de la différence hiérarchique.

A. Desille et C. Le Petitcorps: Dans le projet que tu mènes actuellement, la plantation apparaît comme étant le site central de la production de l'idée de race. Peux-tu, à partir de tes cas d'étude, montrer concrètement comment ce système de production a façonné des catégories et des hiérarchies raciales distinguant les individus et leur place dans la société?

C. Bastos : Comme l'a souligné Edgar Thompson ${ }^{16}$ dans les années 1930 à propos du sud des États-Unis, la plantation est une machine à produire la race. Le sociologue se référait principalement à la manière dont la plantation établit des inégalités radicales, auxquelles se rattachent des catégories cognitives qui hiérarchisent la différence. L'impact de l'esclavage d'Africains était et reste gigantesque, en ce sens qu'il a naturalisé l'inégalité sur laquelle la production était fondée dans le système de plantation. Celle-ci reposait sur un travail inhumain, exécuté par des personnes déshumanisées et traitées comme des marchandises, le tout dans une complicité sereine avec des principes religieux censés bannir ces traitements inhumains. Les théories scientifiques qui justifiaient ces hiérarchies ne manquaient pas, ce qui nous a laissé un héritage contrariant, surtout en anthropologie physique. Les typologies créées par le racialisme ont été reproduites dans de multiples déploiements de sous-types et de sous-sous-types qui ont alimenté des politiques eugéniques, de sélection des migrants, etc.

Le projet de recherche The Colour of Labour approfondit le lien plantation/racialisation en étudiant plusieurs cas qui n'impliquent pas l'asservissement des Africains aux Amériques, mais qui explorent plutôt la mobilisation d'engagés - d'Asie du Sud, de Chine, du Japon, des îles portugaises de l'Atlantique, etc. - dans les sociétés d'après les abolitions de I'esclavage (Guyana, Île Maurice, São Tomé-et-Principe) ou dans les sociétés qui ont adhéré à l'économie de plantation au XIX siècle sans passer par l'esclavage (comme à Hawaï). Plusieurs cas contemporains d'agro-production industrielle qui ont recours au travail

16. THOMPSON, Edgar Tristram, The plantation, op. cit. 
précaire transnational sont aussi abordés, ainsi que le cas de «plantation » mécanique que représente l'industrie textile et son appétit inassouvi pour les masses de travailleurs migrants ${ }^{17}$. Dans ces contextes également, on observe une production de catégories racialisées avec des hiérarchies sous-jacentes ; même si elles s'ouvrent à la dynamique de substitution d'un groupe par un autre, ces catégories collent à la peau, se naturalisent, deviennent un ancrage négatif de l'identification de groupe, une source de malaise et de faible estime de soi collective, avec tout ce que cela implique comme cela a été largement décrit, et quantifié, par les études de psychologie sociale.

A. Desille et C. Le Petitcorps: Vois-tu ce processus de racialisation anciens influencer durablement la position et la construction identitaire des Portugais dans les sociétés de plantation qu'ils ont habitées?

C. Bastos : «Portugee », «Potogee » ou «podagee » sont des termes qui, par le passé, classaient péjorativement les Portugais dans les sociétés de plantation (Hawaï, Guyana) et aussi dans les pôles industriels ou agro-industriels des États-Unis (en Nouvelle-Angleterre notamment). Mais aujourd'hui, leur utilisation ironique ou contreoffensive est rare : la tendance est plutôt de les remplacer par les termes de Portugais, Portugais-Américains ou Portugais de Guyana par exemple, pour marquer une mobilité sociale et économique. Ce dépassement et cette mobilité ont opéré selon différentes modalités. II n'y a pas une «essence » portugaise derrière Potogee ou les Portugais, ces catégories sont le produit de dynamiques locales qui sont singulières à chaque cas. En Guyane britannique, les premiers migrants madériens sont venus remplacer les Africains rendus en esclavage aux mêmes fonctions. Ils ont également fait concurrence aux Afro-descendants dans certaines niches de commerce, ce qui a créé des tensions qui étaient souvent résolues par les hiérarchies établies qui favorisaient les Blancs et s'étendaient aux «quasi-blancs». Les migrants arrivés par la suite lors d'une deuxième vague se trouvèrent déjà dans de meilleures conditions socioéconomiques, puisqu'une communauté portugaise puissante avait été créée dans la colonie britannique. Mais à aucun moment, ni même dans le Guyana actuel où il n'y a presque plus de

17. Ces terrains ont également été étudiés dans le cadre de ce projet par une équipe de recherche pluridisciplinaire : I'historien Nicholas Miller pour le cas de Hawaï, I'historienne des sciences Marta Macedo pour São Tomé-et-Principe, l'anthropologue Irene Peano pour I'agro-production industrielle en Italie, I'anthropologue Marcelo Moura Mello et le géographe João Sardinha pour le Guyana, I'anthropologue Miguel Moniz pour le cas des Portugais dans I'industrie textile en Nouvelle-Angleterre, la sociologue Colette Le Petitcorps et la doctorante en anthropologie Rita Kantu pour l'île Maurice. Pour en savoir plus sur l'équipe, voir le site internet du projet ERC : http://colour.ics.ulisboa.pt/research/tracks/. 
Portugais, la catégorie «Portugais» n'a fusionné avec celle des «Blancs » qui était réservée aux propriétaires de plantations, généralement britanniques ou néerlandais. Les Portugais constituent une catégorie à part, l'une des «six races » constitutives de la nation. II se passe quelque chose de similaire à Hawaï en ce qui concerne la distinction entre « Portugais » et « Haole » (Blanc), marquée par la position initiale du travailleur opposé au patron. Mais la période classique de la plantation qui vivait du travail engagé à l'étranger où les Chinois, les Japonais, les Portugais et plus tard les Coréens étaient maintenus ségrégués et soumis à des hiérarchies qui changeaient au gré des nouvelles conjonctures politiques, a fait place à une autre phase de la plantation avec l'annexion d'Hawaï par les États-Unis, où les travailleurs ont principalement été recrutés à Porto Rico et aux Philippines. De nombreux Portugais ont alors occupé des emplois urbains ou industriels, ont commencé à avoir leur propre production agricole ou bovine ou encore ont occupé des postes de contremaîtres, de superviseurs de travail (dits lunas). Jusqu'en 1930, ils étaient considérés dans le recensement comme un groupe distinct. Après cela, à l'instar des autres groupes à Hawaï, la plupart des familles ont conservé un sentiment d'appartenance et d'ancestralité au groupe qui se combine à beaucoup d'autres appartenances.

A. Desille et C. Le Petitcorps: D'un point de vue méthodologique et à partir des difficultés que tu as toi-même rencontrées, quels conseils pourrais-tu donner aux jeunes chercheuses et chercheurs pour adopter une perspective décoloniale de l'histoire et de l'anthropologie des migrations?

C. Bastos: Je pense que c'est le même ensemble de principes qui devrait être utilisé en toutes circonstances : avoir tous les sens en éveil; se concentrer sur les sujets, en gardant une attitude d'écoute/d'observation/d'attention ethnographique, même lorsqu'il s'agit de sujets disparus depuis longtemps ; croiser les sources ; lire beaucoup de sources diverses ; lire de la littérature locale, académique et de fiction, toujours; prendre ses intuitions au sérieux, mais aussi les tester ; maintenir un scepticisme sain et suspendre notre incrédulité face à des logiques très différentes des nôtres; dialoguer avec les sujets de recherche sur un pied d'égalité ; apprendre des universitaires locaux, sans toutefois projeter sur eux un potentiel de rédemption décoloniale absolue ; ne pas trop généraliser à partir de peu, et essayer d'atteindre une certaine simplicité dans l'écriture. Chercher des sources en dehors du courant dominant et ne pas prendre pour acquises toutes les théories établies. Penser à des lecteurs différents, académi- 
ques et non académiques. Laisser les archives plus accessibles encore que lorsque vous les avez trouvées. Permettre à vos interlocuteurs d'être encore plus disponibles pour le prochain chercheur que lorsque vous les avez rencontrés. 